\title{
LEIA \\ A reverse engineering form for Multi Agent Systems
}

François Gaillard and Yoann Kubera and Philippe Mathieu and Sébastien Picault

SMAC team - LIFL - USTL

UMR CNRS USTL 8022

Villeneuve d'Ascq
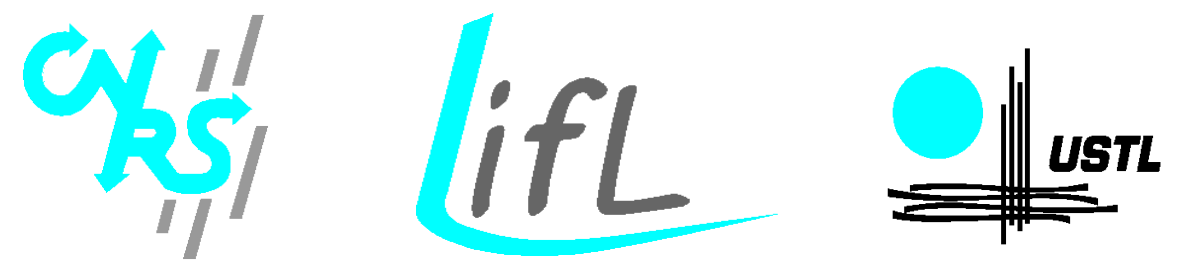


\section{Introduction}

What do we call reverse engineering for MAS ?

Table of contents

I. SMAC team

II. IODA

III. LEIA

IV. An interesting result from $L E I A$

V. Conclusion 


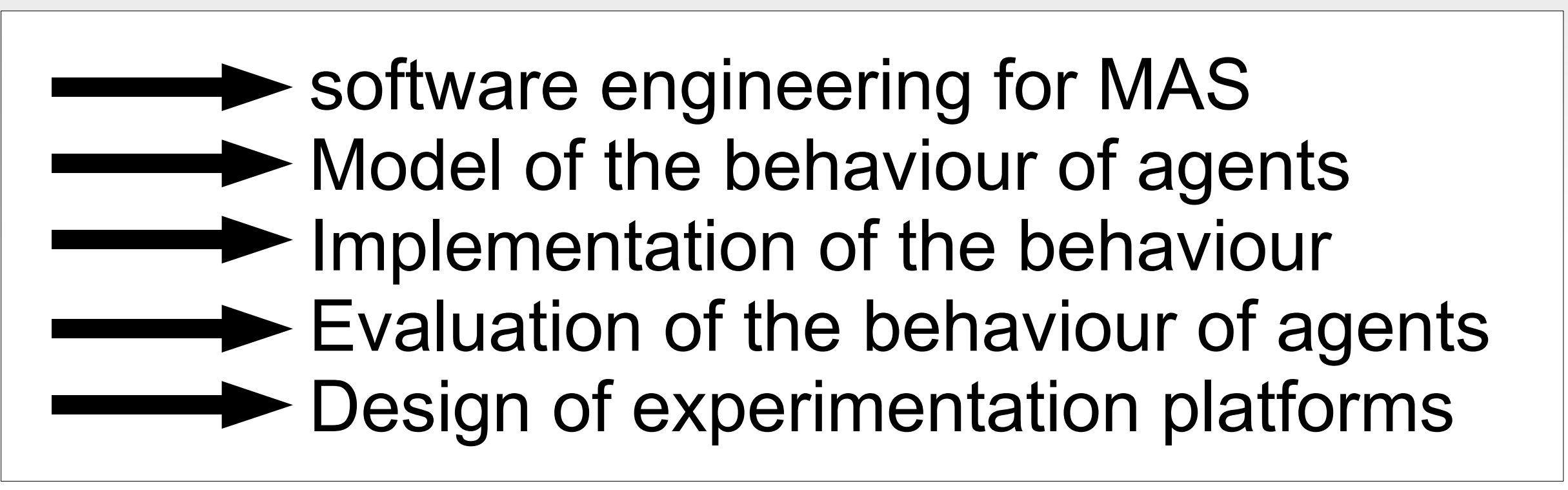

\section{URL: www.lifl.fr/SMAC}




\section{Interaction Oriented Design of Agent simulation}

\section{Methodology centered on Interactions}

[Mathieu Routier 01]

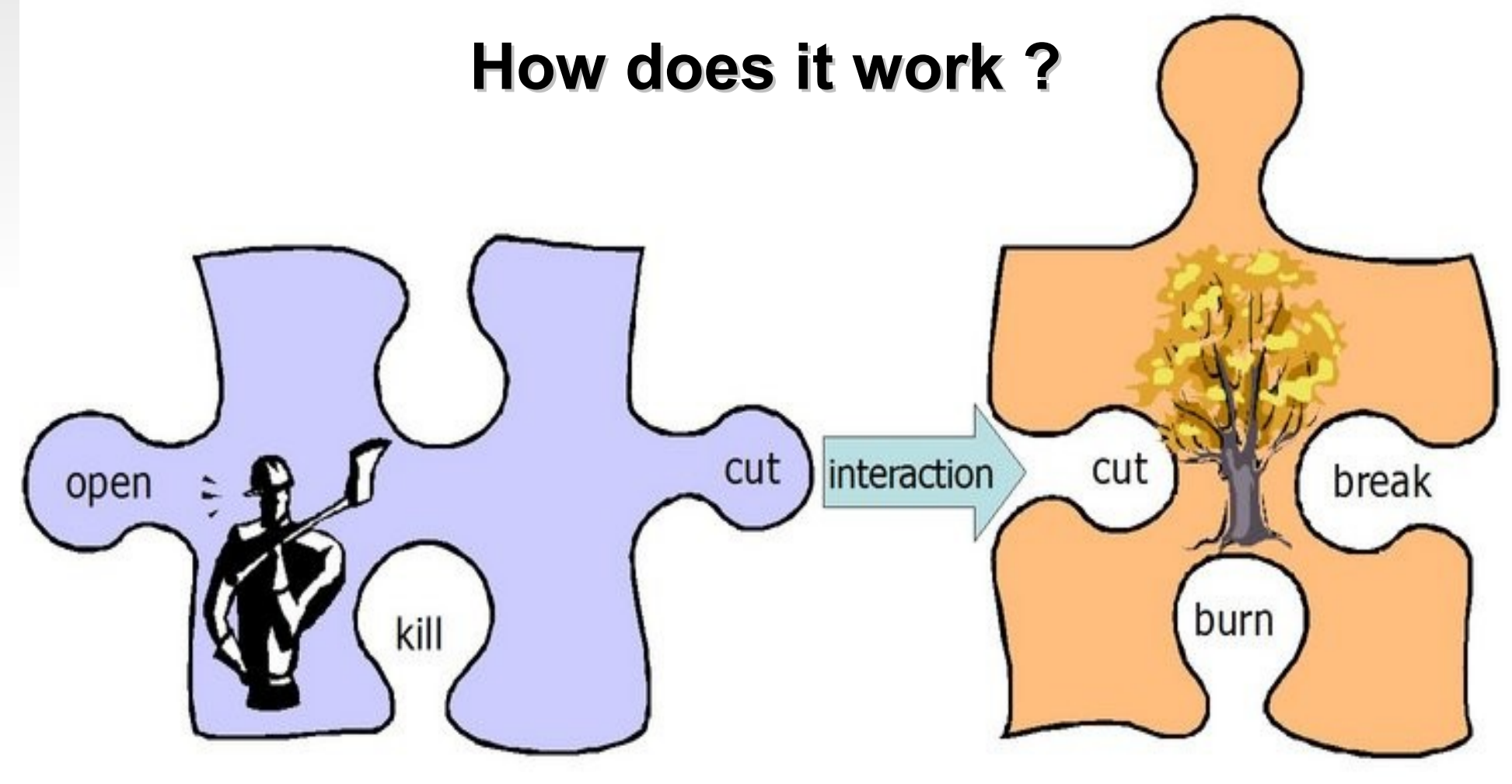

Separation between what an agent can do and how he will do it 


\section{How can an interaction occur? [Kubera 08]}

Declarative part

$\rightarrow$ Interaction

$\rightarrow$ Source/Target

$\rightarrow$ Distance Guard

\section{Procedural part}

$\rightarrow$ Order in the evaluation of the interactions

$\rightarrow$ Selection process

\section{Interaction Matrix}

\begin{tabular}{|c|c|c|c|}
\hline Source $\quad$ Target & Envir. & Red & Blue \\
\hline Red & & Follow $0(4.0)$ & Run 2(7.0) \\
\hline Blue & & $(5.0)$ & Follow $1(2.0)$ \\
\hline
\end{tabular}




\section{III - LEIA: the exploration of simulations as a reverse engineering form}

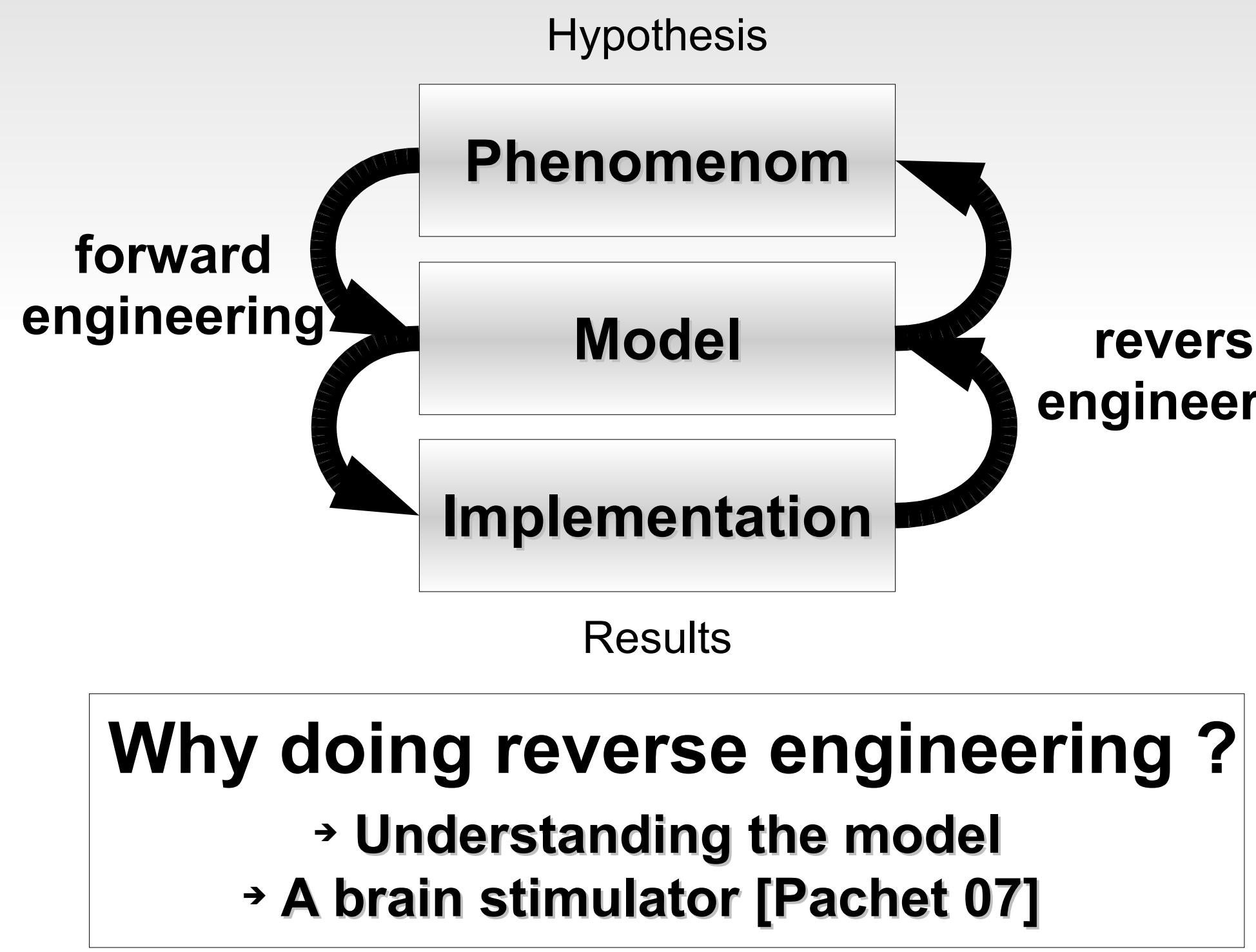




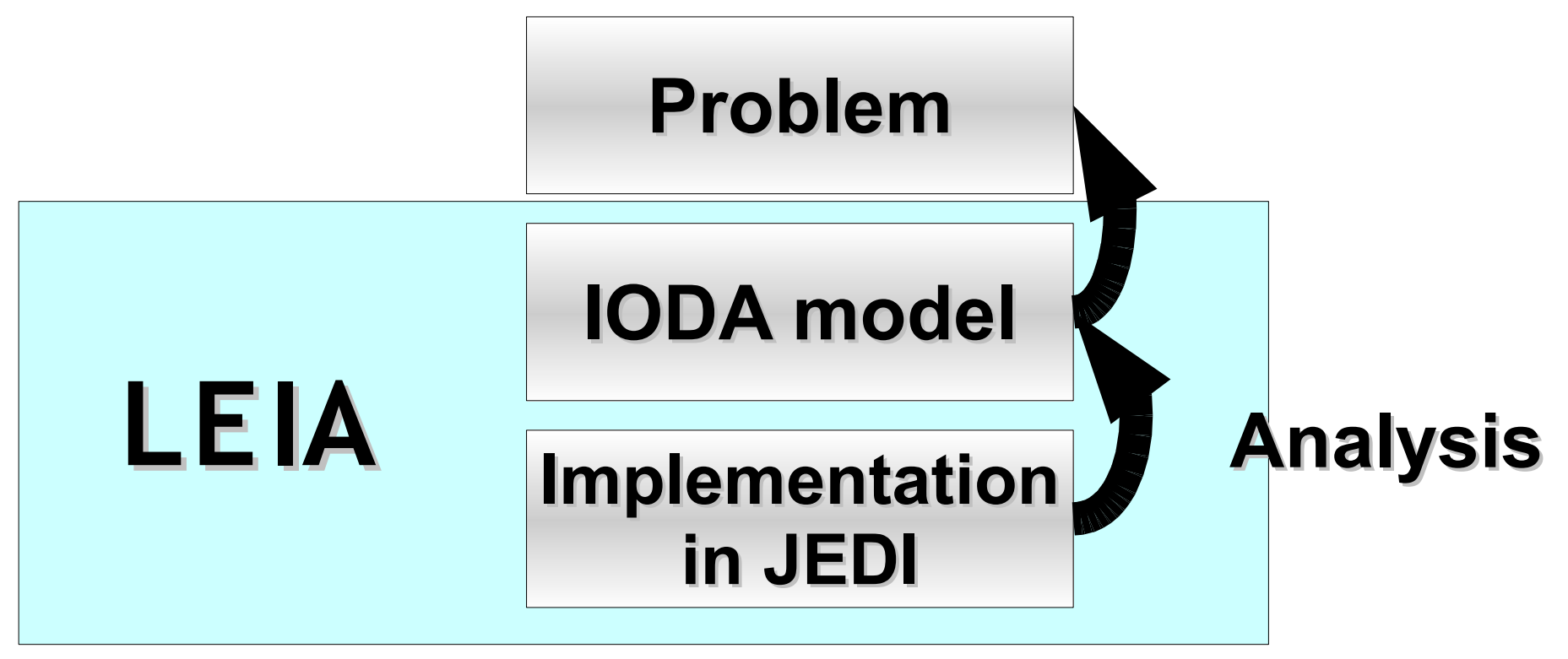

$\rightarrow$ Reverse engineering on simulations

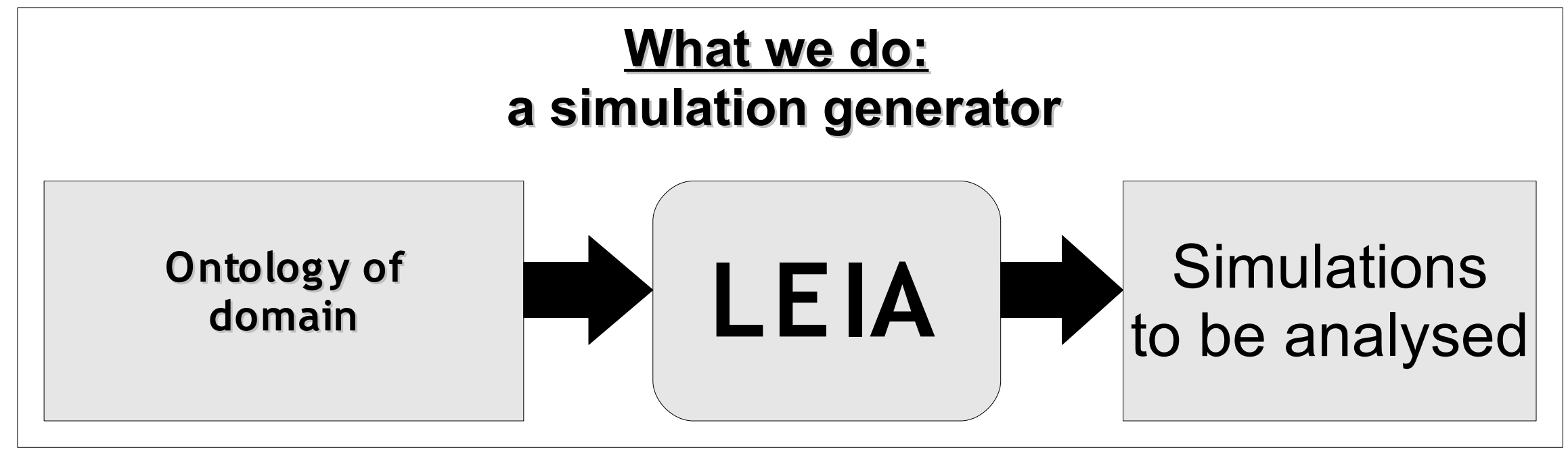


III - LEIA - Automatized building of model

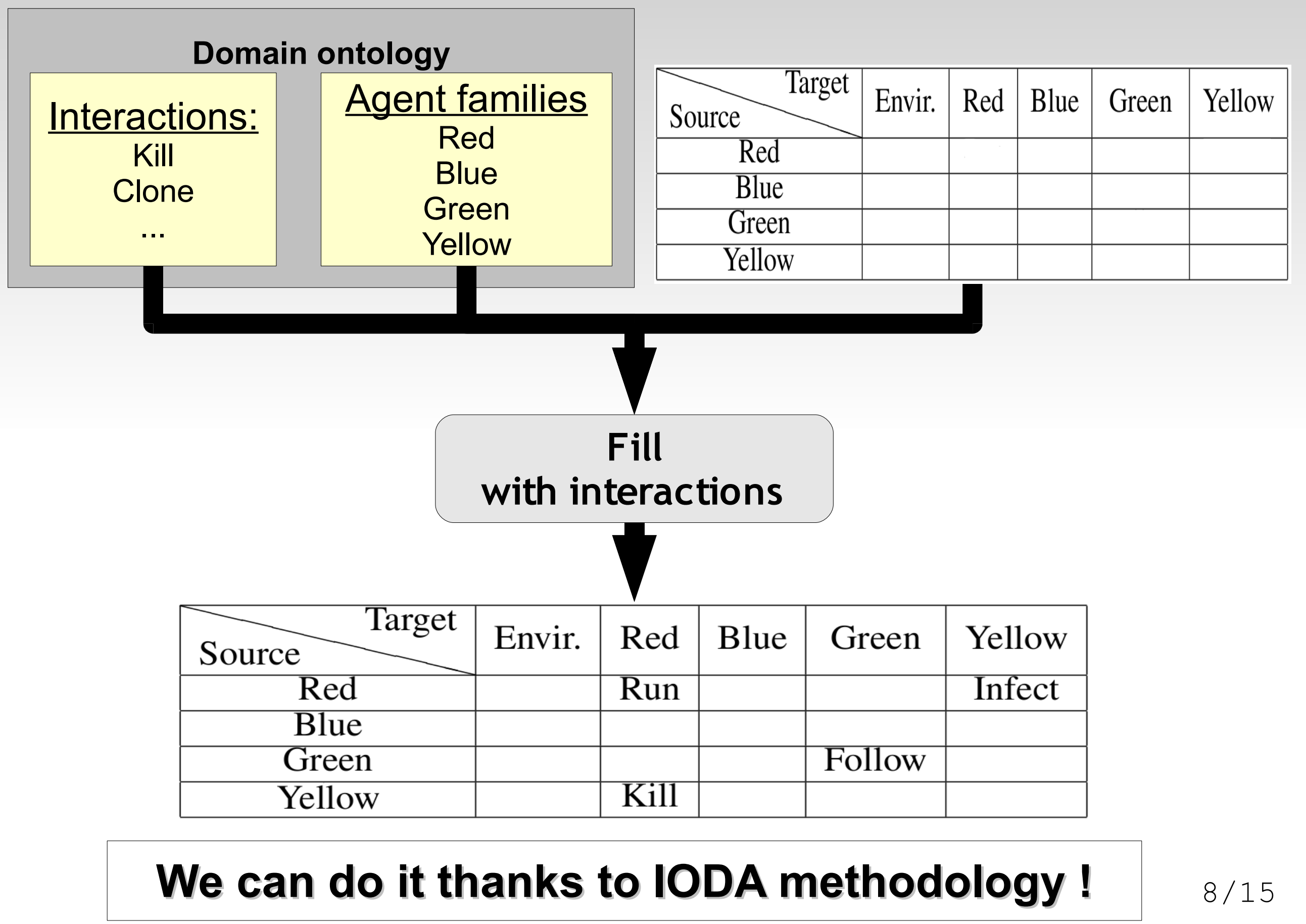




\section{III - LEIA - Let's explore the simulations space}

Iterative construction of models by exploring the simulations space

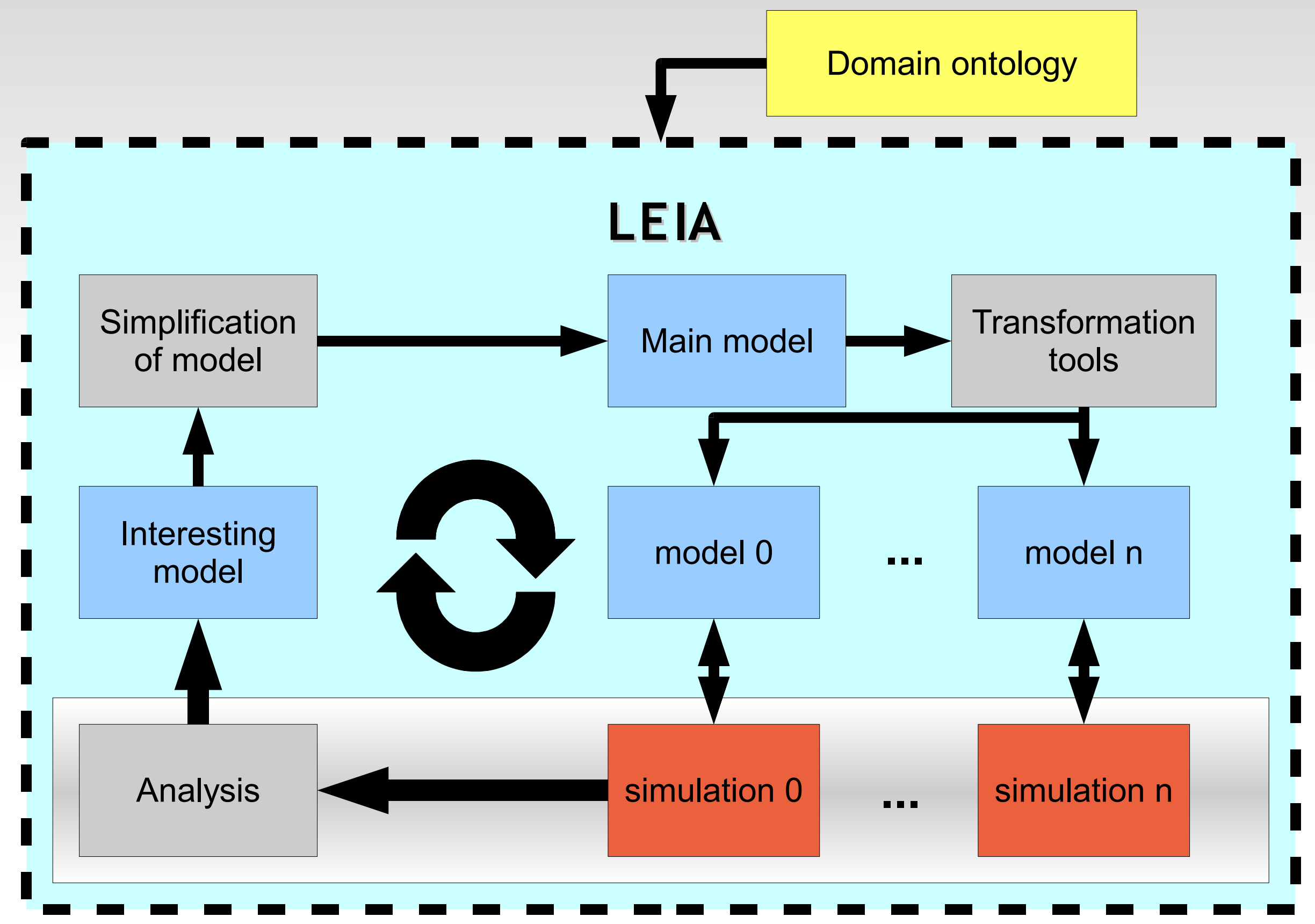




\section{III - LEIA - our measurement tools}

Our aim: analysing a simulation during the runtime

Activity of the agents

The evolution of the population

Density of population
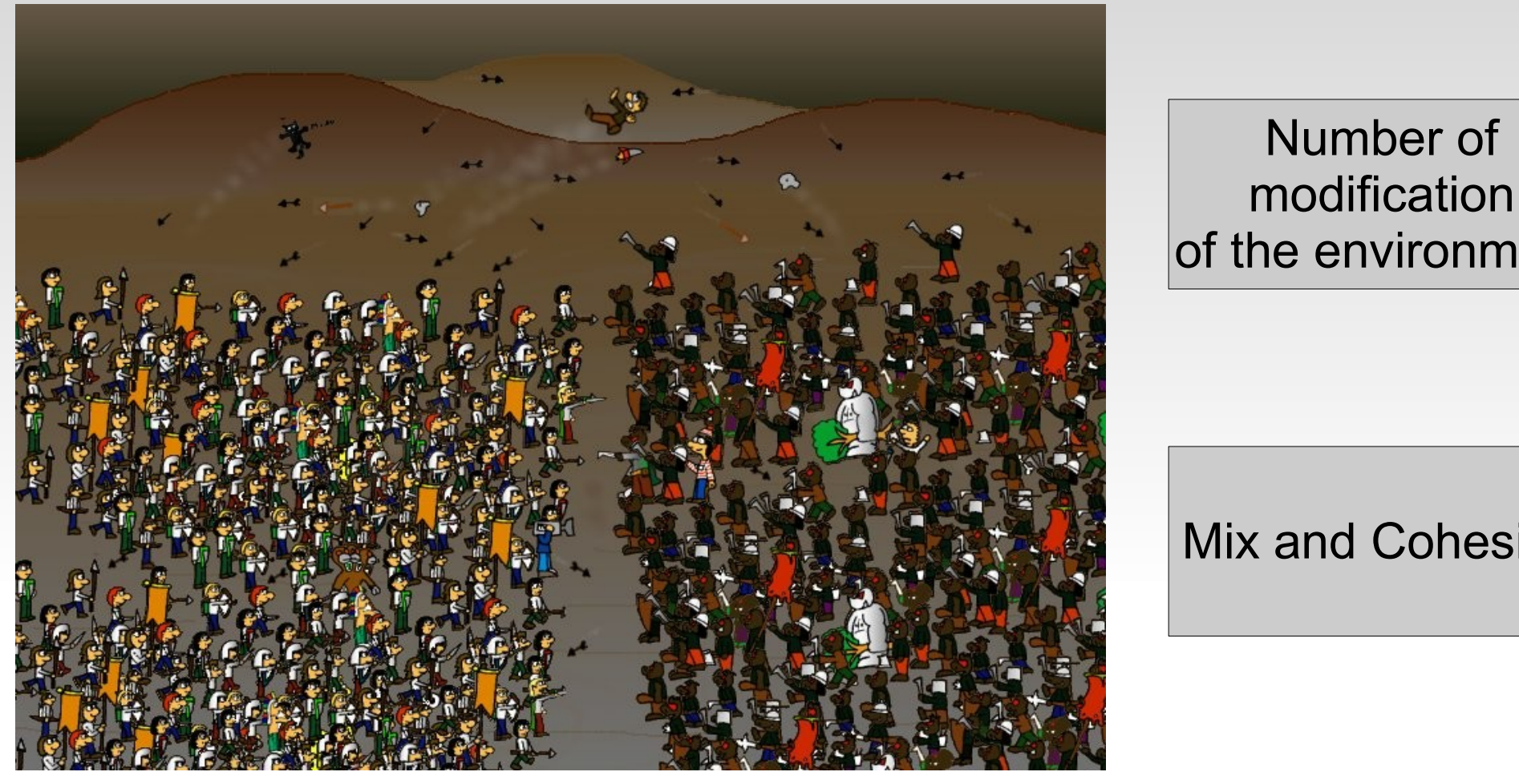
modification of the environment

Mix and Cohesion

\section{An example: a battle simulation}

\section{Main points:}

$\rightarrow$ Using the separation Interactions/Agents

$\rightarrow$ Working with any JEDI simulation and any ontology of domain

$\rightarrow$ Data from each families of agent and interactions 


\section{Our aims:}

$\rightarrow$ Find new models

$\rightarrow$ Test the robustness of existing models

$\rightarrow$ Test an ontology of domain

\section{Our model transformation tools:}

$\rightarrow$ Test on the beginning number of agent

$\rightarrow$ Add/Remove random interactions

$\rightarrow$ Modification of the distance guard/priority

$\rightarrow$ Derivation of an interesting model into new models

$\rightarrow$ Play with several interaction matrices at a time 


\section{III - LEIA - Let's explore the simulations space}

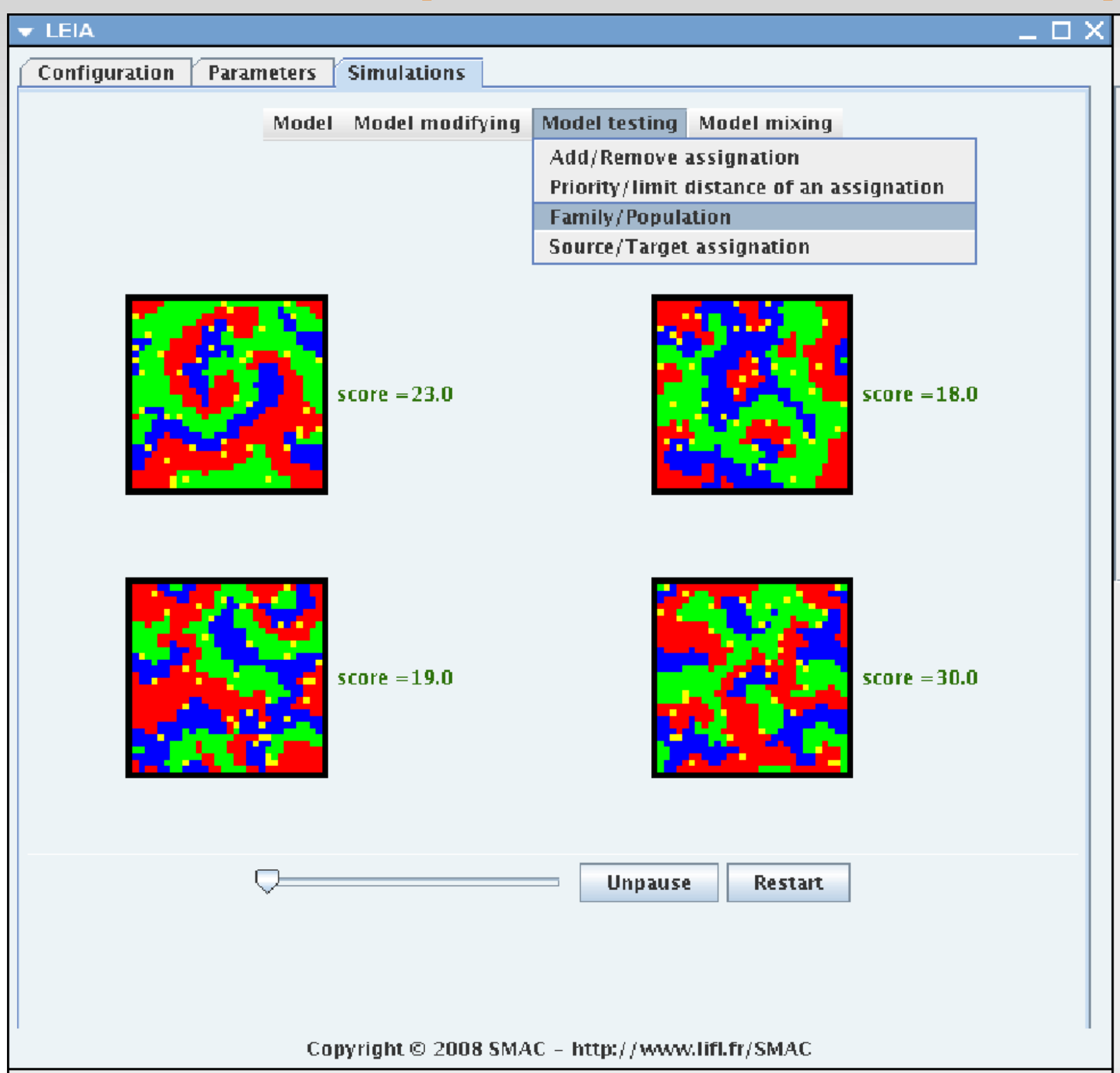




\section{IV - An interesting result from LEIA}

\section{an infection model}

\begin{tabular}{|c|l|l|l|l|l|}
\hline Source Target & Envir. & Red & Blue & Green & Yellow \\
\hline Red & & & Infect & & \\
\hline Blue & & & & Infect & \\
\hline Green & & & & & Infect \\
\hline Yellow & & Infect & & & \\
\hline
\end{tabular}

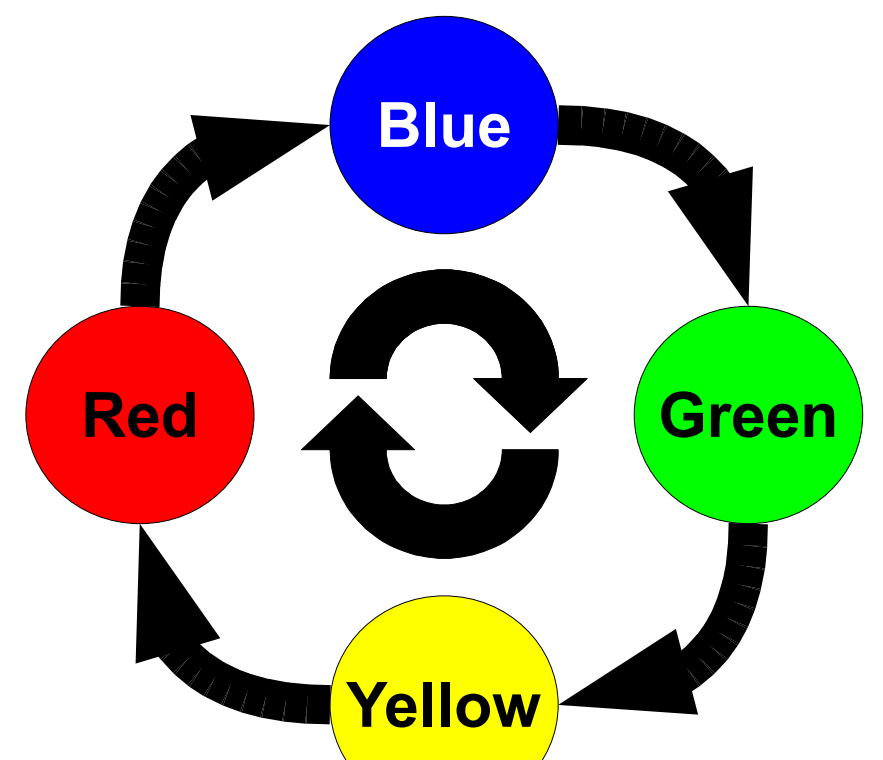

\section{Excitable medium}

Cyclical cellular automaton [Griffeath 93]

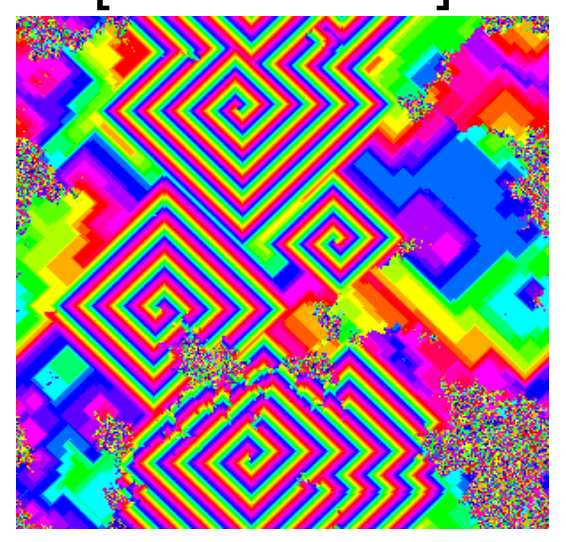

Belousov-Zhabotinsky reaction [Belousov 59, Zhabotinsky 50]
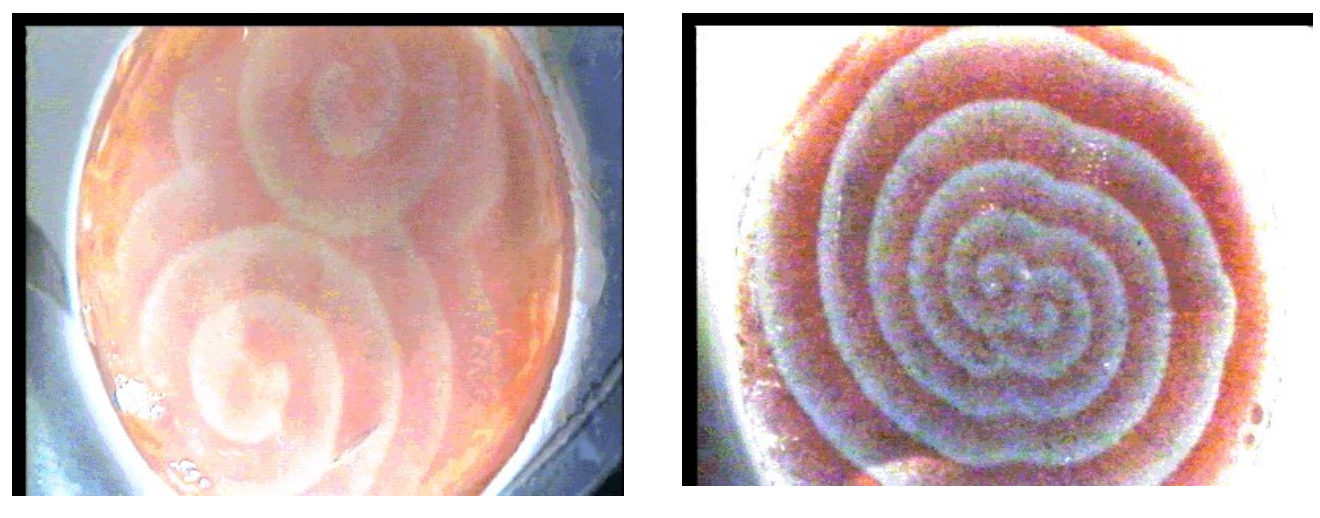
$\rightarrow$ A simulation generator without any code generation

$\rightarrow$ Made thanks to IODA methodology

$\rightarrow$ The user is implied in the iterative process of exploration of the simulations space in order to create new models

$\rightarrow$ guide lines to improve simulations following several metrics

$\rightarrow$ Reverse engineering by analysis of simulations

$\rightarrow$ Take place in a whole framework from the conception of interactions to their implementations using IODA, JEDI, JEDI Builder and now LEIA

$\rightarrow$ Exploration of an ontology of domain with genetic algorithm

\section{Let's visit www.lifl.fr/SMAC/LEIA/}




\section{Bibliography}

$\rightarrow$ [Philippe Routier 01] Philippe Mathieu and Jean-Christophe Routier and Pascal Urro Un modèle de simulation agent basé sur les interactions

$\rightarrow$ [Pac07] F. Pachet. De la co-construction d'un langage homme-machine : quelques expériences en musique (JFSMA'2007)

$\rightarrow$ [Kubera 08] Sébastien Picault Yoann Kubera, Philippe Mathieu. Interaction-oriented agent simulations :From theory to implementation, ECAl 08 July 21-25 2008.

$\rightarrow$ [Kubera 08] Sébastien Picault Yoann Kubera, Philippe Mathieu. Une architecture orientée interactions. Revue d'Ingéniérie des Systèmes d'Information (ISI), 2008.

$\rightarrow$ [FGG93] R. Fisch, J. Gravner, and D. Griffeath. Metastability in the Greenberg-Hastings Model. March 1993.

$\rightarrow$ [Bel59] B. P. Belousov. A periodic reaction and its mechanism. In Compilation of Abstracts on Radiation Medicine, 1959.

$\rightarrow$ [Zha64] A. M. Zhabotinsky. Periodic processes of malonic acid oxidation in a liquid phase. In Biofizika, 1964.

$\rightarrow$ [Holland 75] Adaptation in natural and artificial systems

$\rightarrow$ [MonMarché Venturini 99] Imagine : a tool for generating HTML style sheets with an interactive genetic algorithm based on genes frequencies 